\title{
Gamma rays from Fast Black-Hole Winds
}

\section{Chris Karwin, ${ }^{a, *}$ Marco Ajello, ${ }^{a}$ Rebecca Diesing, ${ }^{b}$ Damiano Caprioli ${ }^{b}$ and George \\ Chartas $^{c}$ on behalf of the Fermi-LAT Collaboration \\ (a complete list of authors can be found at the end of the proceedings)}

${ }^{a}$ Department of Physics and Astronomy, Clemson University,

Clemson, SC, 29634 USA

${ }^{b}$ Department of Astronomy and Astrophysics, University of Chicago,

Chicago, IL, 69637, USA

${ }^{c}$ Department of Physics and Astronomy, College of Charleston,

Charleston, SC, 29424, USA

E-mail: ckarwin@clemson.edu, majello@clemson.edu, caprioli@uchicago.edu,

rrdiesing@uchicago.edu, chartasg@cofc.edu

Massive black holes at the centers of galaxies can launch powerful wide-angle winds that, if sustained over time, can unbind the gas from the stellar bulges of galaxies. These winds may be responsible for the observed scaling relation between the masses of the central black holes and the velocity dispersion of stars in galactic bulges. Propagating through the galaxy, the wind should interact with the interstellar medium creating a strong shock, similar to those observed in supernovae explosions, which is able to accelerate charged particles to high energies. In this work we use data from the Fermi Large Area Telescope to search for the $\gamma$-ray emission from galaxies with an ultra-fast outflow (UFO): a fast $(v \sim 0.1 \mathrm{c})$, highly ionized outflow, detected in absorption at hard X-rays in several nearby active galactic nuclei (AGN). Adopting a sensitive stacking analysis we are able to detect the average $\gamma$-ray emission from these galaxies and exclude that it is due to processes other than the UFOs. Moreover, our analysis shows that the $\gamma$-ray luminosity scales with the AGN bolometric luminosity and that these outflows transfer $\sim 0.04 \%$ of their mechanical power to $\gamma$ rays. Interpreting the observed $\gamma$-ray emission as produced by cosmic rays (CRs) accelerated at the shock front, we find that the $\gamma$-ray emission may attest to the onset of the wind-host interaction and that these outflows can energize charged particles up to the transition region between galactic and extragalactic CRs. A preprint of the full analysis is available on the arXiv: 2105.11469 .

$37^{\text {th }}$ International Cosmic Ray Conference (ICRC 2021)

July 12th - 23rd, 2021

Online - Berlin, Germany

\footnotetext{
${ }^{*}$ Presenter
} 


\section{Introduction}

A fraction of the energy accumulated in active galactic nuclei (AGN) during their accretion phase can be released via the ejection of powerful outflows driven by the AGN itself, i.e. blackhole winds. It is thought that these outflows may contribute significantly to galaxy evolution and feedback, namely, the evolution of the bulge, the star formation, and the black hole growth [1]. Among the different types of outflows, the most powerful non-collimated ones are the so-called ultra fast outflows (UFOs). Primarily identified through X-ray observations of blue-shifted Fe K-shell absorption lines [1-8], UFOs are characterized by highly ionized gas with large column densities and mildly relativistic velocities (mean velocity of $\sim 0.1 \mathrm{c}$ ). They are located at sub-parsec scales from the central black hole of the host galaxy, and they have a mass outflow rate of $\sim 0.01-1 \mathrm{M}_{\odot} \mathrm{yr}^{-1}$, corresponding to a high kinetic power of $\log \dot{E}_{\mathrm{k}} \sim 42-45 \mathrm{erg} \mathrm{s}^{-1}$ [1]. It has been predicted that the outflowing gas of a UFO may generate shock waves through interactions with the surrounding interstellar medium [9-11]. Due to the large kinetic power involved, this process may accelerate electrons and protons to high energies, subsequently generating non-thermal $\gamma$-ray emission, similar to what occurs with supernova remnants. For this reason it has been predicted that UFOs may be $\gamma$-ray emitters [9-11].

In this work we search for the $\gamma$-ray emission from UFOs using data collected by the Large Area Telescope [LAT 12] on board the Fermi Gamma-ray Space Telescope [12]. Models of the $\gamma$-ray emission from AGN outflows $[9,11]$ show them to be weak emitters, with $\gamma$-ray luminosities of $\approx 10^{40} \mathrm{erg} \mathrm{s}^{-1}$, which explains why UFOs have not yet been detected by the LAT. Here, we adopt a different strategy and search for the collective $\gamma$-ray emission from a sample of UFOs using a stacking technique.

\section{Sample Selection and Stacking Technique}

We start from a sample of 35 sources that have been identified as UFOs through X-ray observations [2-6]. We have verified that none of the objects are positionally coincident with any known $\gamma$-ray sources reported in the 4FGL [13]. From the initial sample we make the following cuts. First, we only keep the radio-quiet sources (as specified in the original references) to avoid contamination of the signal from the relativistic jet. Furthermore, we only select sources that are nearby $(z<0.1)$ with a mildly relativistic wind velocity $(v>0.1 c)$. The former cut is motivated by the expected low luminosity of the UFO emission [9], and the latter cut is motivated by the fact that the $\gamma$-ray emission is predicted to scale with the kinetic power of the outflow $[9,11]$. After making these cuts we are left with 11 sources, which we use as our benchmark sample.

The stacking technique we employ has been developed previously and has been successfully employed for multiple studies, i.e. upper limits on dark matter interactions [14], detection of the extragalactic background light [15], extreme blazars [16], and star-forming galaxies [17]. The main assumption that we make for the stacking technique is that the sample of UFOs we are considering can be characterized by average quantities like the average flux and the average photon index (when we model their spectra with a power law). There are then two steps to the method. In the first step the model components are optimized for each ROI using a maximum likelihood fit. We evaluate the significance of each source in the ROI using the TS, which is defined as TS $=-2 \log \left(\mathrm{L}_{0} / \mathrm{L}\right)$, where 

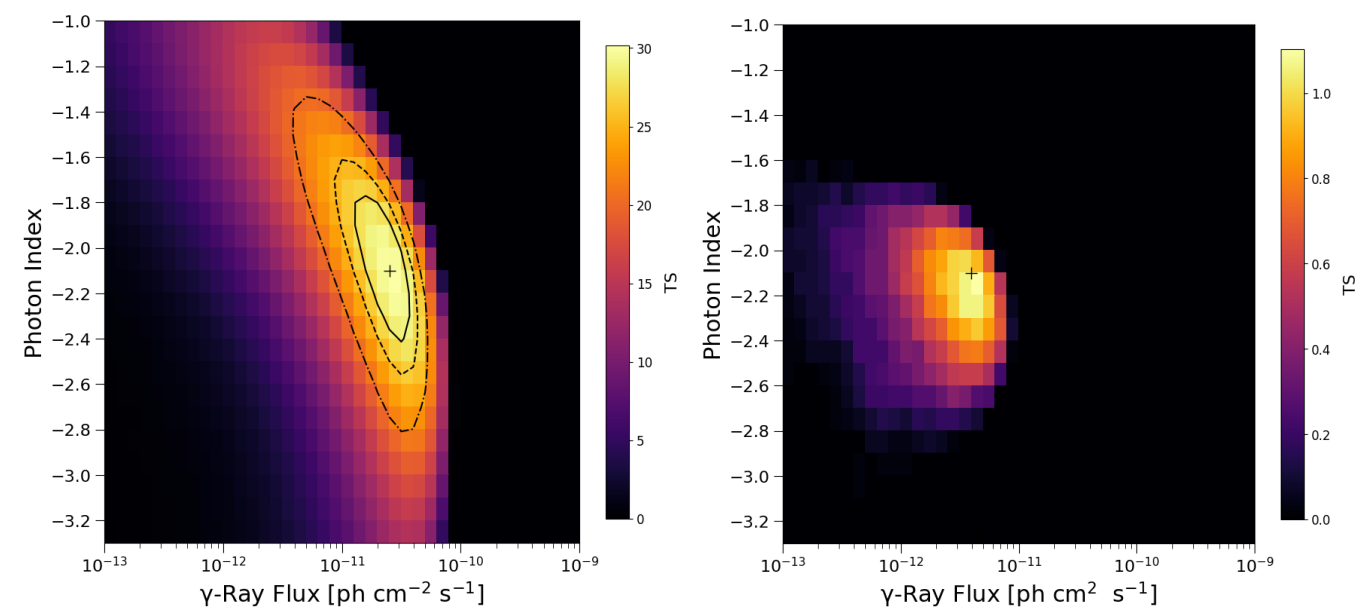

Figure 1: Left: Stacked TS profile for the sample of UFOs. The color scale indicates the TS, and the plus sign indicates the location of the maximum value, with a TS $=30.1(5.1 \sigma)$. Significance contours (for 2 degrees of freedom) are overlaid on the plot showing the $68 \%, 90 \%$, and $99 \%$ confidence levels, corresponding to $\Delta \mathrm{TS}=2.30,4.61$, and 9.21, respectively. Right: Stacked profile for our control sample consisting of 20 nearby $(z<0.1)$ radio-quiet AGN with no UFOs (i.e. a UFO has been searched for but none has been detected). No signal is detected, with a maximum TS of 1.1.

$\mathrm{L}_{0}$ is the likelihood for the null hypothesis, and $\mathrm{L}$ is the likelihood for the alternative hypothesis. For the first iteration of the fit, the spectral parameters of the Galactic diffuse component (index and normalization) and the isotropic component are freed. In addition, we free the normalizations of all 4FGL sources with $\mathrm{TS} \geq 25$ that are within $5^{\circ}$ of the ROI center, as well as sources with $\mathrm{TS} \geq 500$ and within $7^{\circ}$. Lastly, the UFO source is fit with a power-law spectral model, and the spectral parameters (normalization and index) are also freed. In the first step we also find new point sources using the Fermipy function find_sources, which generates TS maps and identifies new sources based on peaks in the TS. The TS maps are generated using a power-law spectral model with an index of -2.0 . The minimum separation between two point sources is set to $0.5^{\circ}$, and the minimum TS for including a source in the model is set to 16 .

In the second step 2D TS profiles are generated for the spectral parameters of each UFO source. We scan photon indices from -1 to -3.3 with a spacing of 0.1 and total integrated photon flux (between $1-800 \mathrm{GeV}$ ) from $10^{-13}$ to $10^{-9} \mathrm{ph} \mathrm{cm}^{-2} \mathrm{~s}^{-1}$ with 40 logarithmically spaced bins, freeing just the parameters of the diffuse components. Lastly, the TS profiles for all sources are added to obtain the stacked profile. The TS is an additive quantity, and so the stacked profile gives the statistical significance for the combined signal.

\section{Results}

The stacked profile for our UFO sample is shown in the left panel of Figure 1. The maximum TS is $30.1(5.1 \sigma)$, corresponding to a best-fit index of $-2.1 \pm 0.3$ and a best-fit photon flux (1-800 $\mathrm{GeV}$ ) of $2.5_{-0.9}^{+1.5} \times 10^{-11} \mathrm{ph} \mathrm{cm}^{-2} \mathrm{~s}^{-1}$. The $68 \%, 90 \%$, and $99 \%$ significance contours are overlaid on the map, and as can be seen the spectral parameters are well constrained. 


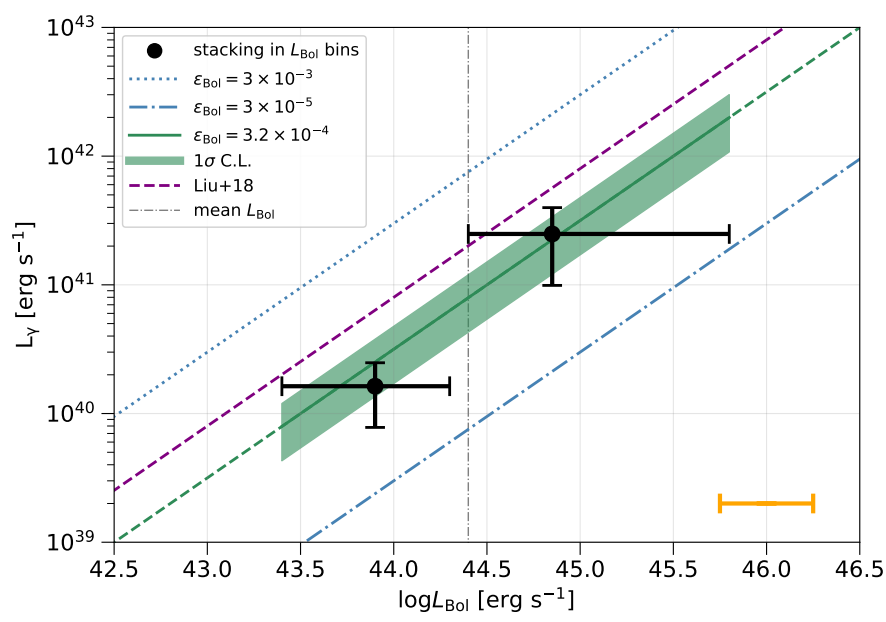

Figure 2: $\gamma$-ray luminosity versus bolometric luminosity. The black data points result from stacking in $\gamma$-ray luminosity, and the uncertainty in the $\mathrm{x}$-axis corresponds to the bin widths. The grey dash-dot vertical lines show the value used to divide the bins. The solid green line shows the best-fit resulting from stacking in efficiency, with the green band showing the $1 \sigma$ confidence level. For reference, the blue lines show a range of efficiencies within roughly an order of magnitude of the best fit. The orange bar shows the average onesided uncertainty in individual measurements of AGN bolometric luminosity. We also overlay the predicted efficiency derived from [19, dashed purple line].

We repeat the analysis with a sample of 20 low redshift $(z<0.1)$ radio-quiet AGN that do not have UFOs. The sources were selected from the samples of [4] and [18] for which no UFO was found. The sample of [4] is based on absorption features, while the sample of [18] uses the excess variance method. Of the 20 sources in our control sample, there are 10 sources in common between the two studies, 4 additional sources from [4], and 6 additional sources from [18]. Results for the stacked profile are shown in the right panel of Figure 1. No signal is detected, with a maximum TS of 1.1. Using the profile likelihood method and a photon index of -2.0 , the upper limit on the flux $(1-800 \mathrm{GeV})$ at the $95 \%$ confidence level is $8.8 \times 10^{-12} \mathrm{ph} \mathrm{cm}^{-2} \mathrm{~s}^{-1}$. We have also shown that the $\gamma$-ray emission observed in the UFOs is a factor of $\sim 40$ larger than what we would expect for star-formation activity, and that it's highly unlikely the UFO emission results from weak jets. This supports the interpretation of the $\gamma$-ray emission being due to the outflow rather than other processes in AGN.

The $\gamma$-ray luminosity from UFOs is predicted to scale with the AGN bolometric luminosity. To test this relationship we calculate the stacked profile in bins of bolometric luminosity. Additionally, we calculated the stacked profile in terms of efficiency, defined as $\epsilon_{\mathrm{Bol}}=L_{\gamma} / L_{\mathrm{Bol}}$. Results for this are shown in Figure 2. Indeed, we do find a scaling relation, and we measure the best-fit efficiency to be $(3.2 \pm 1.5) \times 10^{-4}$.

\section{Model}

The physical model for the UFO SED is calculated by assuming that the $\gamma$-ray emission is dominated by hadronic processes resulting from diffusive shock acceleration. For typical UFO 

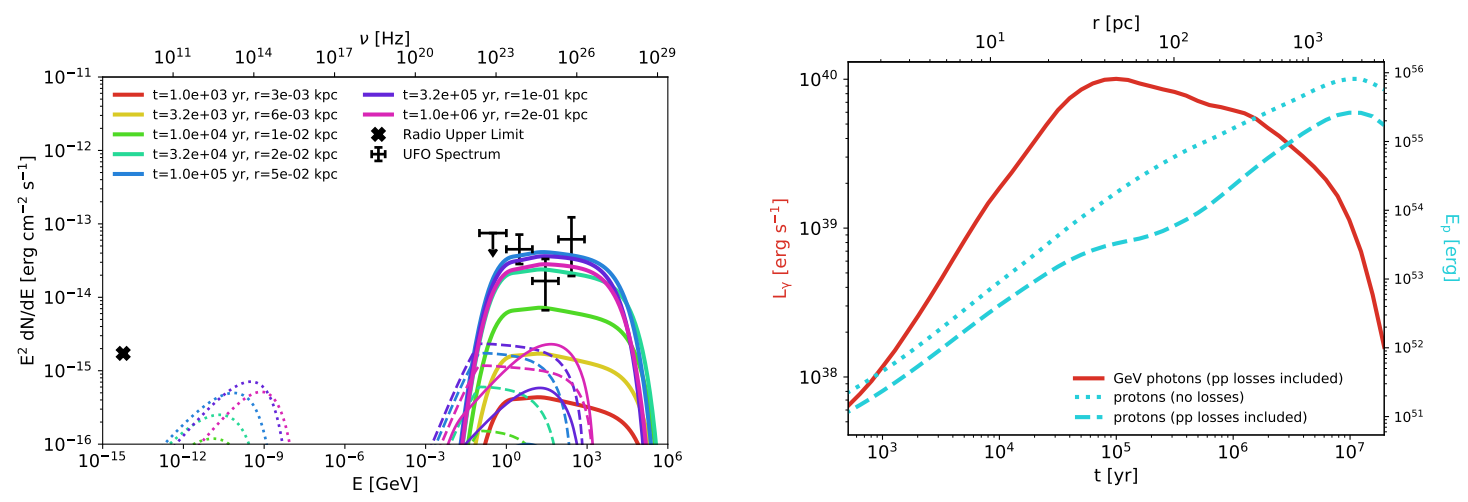

Figure 3: Left: Predicted multiwavelength SED of the UFO's nonthermal emission as a function of time. Synchrotron emission (dotted curves), bremsstrahlung emission (dashed curves), inverse-Compton emission (thin solid curves) and emission from $\pi^{0}$-decay (thick solid curves) are shown. The inverse-Compton emission remains subdominant despite assuming an artificially enhanced stellar radiation field of energy density 100 $\mathrm{eV} \mathrm{cm}^{-3}$. Also overlaid is the observed $\gamma$-ray flux and the average radio upper limit. Note that the leptonic emission produced at early times often does not appear as it falls below the plot range. Right: Light curve of a UFO-powered forward shock moving through a representative galaxy. The total energy in CRs is shown before and after proton-proton losses are included (blue dotted and dashed lines, respectively), as is the $\gamma$-ray luminosity at $1 \mathrm{GeV}$ (red solid line).

shock velocities and densities, a leptonic origin of the $\gamma$-ray emission is disfavored, in that inverseCompton scattering and bremsstrahlung of relativistic electrons would produce steeper $\gamma$-ray spectra with a lower normalization. The observed $\gamma$-ray SED indicates a firm detection of CR protons with energies reaching at least as high as $\approx 10^{12-13} \mathrm{eV}$.

Within our hadronic emission model we derive that on average the forward shock has traveled $\sim 20-300 \mathrm{pc}(\sim 65-980$ light years $)$ away from the SMBH and that the maximum energy of protons accelerated at the forward shock is $\approx 10^{17} \mathrm{eV}$. This makes AGN winds a potential source of CRs with energies beyond the 'knee' of the CR spectrum (i.e., $3 \times 10^{15} \mathrm{eV}$ ) and also likely contributors to the IceCube neutrino flux [20].

\section{Acknowledgments}

M. Ajello and C. Karwin acknowledge support from NSF and NASA through grants AST1715256 and 80NSSC18K1718. R. Diesing and D. Caprioli acknowledge the Eugene \& Niesje Parker Fellowship Fund, NASA (grants NNX17AG30G, 80NSSC18K1218, and 80NSSC18K1726) and the NSF (grants AST-1714658, AST-1909778). G. Chartas acknowledges financial support from NASA grants 80NSSC20K0438 and 80NSSC19K095.

The Fermi-LAT Collaboration acknowledges support for LAT development, operation and data analysis from NASA and DOE (United States), CEA/Irfu and IN2P3/CNRS (France), ASI and INFN (Italy), MEXT, KEK, and JAXA (Japan), and the K.A. Wallenberg Foundation, the Swedish Research Council and the National Space Board (Sweden). Science analysis support in the operations phase from INAF (Italy) and CNES (France) is also gratefully acknowledged. This work performed in part under DOE Contract DE-AC02-76SF00515. 


\section{References}

[1] F. Tombesi, M. Cappi, J. N. Reeves, R. S. Nemmen, V. Braito, M. Gaspari, and C. S. Reynolds. Unification of x-ray winds in seyfert galaxies: from ultra-fast outflows to warm absorbers. MNRAS, 430(2):1102-1117, 2013.

[2] J.N. Reeves, P. T. O'Brien, and M. J. Ward. A massive x-ray outflow from the quasar pds 456. ApJ, 593(2):L65, 2003.

[3] F. Tombesi, R. M. Sambruna, J. N. Reeves, V. Braito, L. Ballo, J. Gofford, M. Cappi, and R. F. Mushotzky. Discovery of ultra-fast outflows in a sample of broad-line radio galaxies observed with suzaku. ApJ, 719(1):700, 2010.

[4] F. Tombesi, M. Cappi, J. N. Reeves, G. G. C. Palumbo, T. Yaqoob, V. Braito, and M. Dadina. Evidence for ultra-fast outflows in radio-quiet agns-i. detection and statistical incidence of fe k-shell absorption lines. $A \& A, 521: A 57,2010$.

[5] F. Tombesi, M. Cappi, J. N. Reeves, and V. Braito. Evidence for ultrafast outflows in radio-quiet agns-iii. location and energetics. MNRAS, 422(1):L1-L5, 2012.

[6] J. Gofford, J. N. Reeves, F. Tombesi, V. Braito, T. J. Turner, L. Miller, and M. Cappi. The suzaku view of highly ionized outflows in agn-i. statistical detection and global absorber properties. MNRAS, 430(1):60-80, 2013.

[7] F. Tombesi, F. Tazaki, R. F. Mushotzky, Y. Ueda, M. Cappi, J. Gofford, J. N. Reeves, and M. Guainazzi. Ultrafast outflows in radio-loud active galactic nuclei. MNRAS, 443(3):21542182, 2014.

[8] J. Gofford, J. N. Reeves, D. E. McLaughlin, V. Braito, T. J. Turner, F. Tombesi, and M. Cappi. The suzaku view of highly ionized outflows in agn-ii. location, energetics and scalings with bolometric luminosity. MNRAS, 451(4):4169-4182, 2015.

[9] X. Wang and A. Loeb. Contribution of quasar-driven outflows to the extragalactic gamma-ray background. NatPh, 12(12):1116, 2016.

[10] A. Lamastra, F. Fiore, D. Guetta, L. A. Antonelli, S. Colafrancesco, N. Menci, S. Puccetti, A. Stamerra, and L. Zappacosta. Galactic outflow driven by the active nucleus and the origin of the gamma-ray emission in ngc 1068. A\&A, 596:A68, 2016.

[11] A. Lamastra, N. Menci, F. Fiore, L. A. Antonelli, S. Colafrancesco, D. Guetta, and A. Stamerra. Extragalactic gamma-ray background from agn winds and star-forming galaxies in cosmological galaxy-formation models. $A \& A, 607: \mathrm{A} 18,2017$.

[12] W. B. Atwood et al. The large area telescope on the fermi gamma-ray space telescope mission. ApJ, 697:1071-1102, 2009.

[13] S. Abdollahi, et al. Fermi Large Area Telescope Fourth Source Catalog. ApJS, 247(1):33, March 2020. 
[14] M. Ackermann, et al. Constraining Dark Matter Models from a Combined Analysis of Milky Way Satellites with the Fermi Large Area Telescope. PhRvL, 107(24):241302, December 2011.

[15] S. Abdollahi et al. A gamma-ray determination of the Universe's star formation history. Sci, 362(6418):1031-1034, 2018.

[16] V. S. Paliya, A. Dominguez, M. Ajello, A. Franckowiak, and D. Hartmann. Fermi-lat stacking analysis technique: An application to extreme blazars and prospects for their cta detection. ApJ, 882(1):L3, 2019.

[17] M. Ajello, M. Di Mauro, V. S. Paliya, and S. Garrappa. The $\gamma$-Ray Emission of Star-forming Galaxies. ApJ, 894(2):88, May 2020.

[18] Z. Igo, M. L. Parker, G. A. Matzeu, W. Alston, N. Alvarez Crespo, F. Fürst, D. J. K. Buisson, A. Lobban, A. M. Joyce, L. Mallick, N. Schartel, and M. Santos-Lleó. Searching for ultra-fast outflows in AGN using variability spectra. MNRAS, 493(1):1088-1108, March 2020.

[19] R. Y. Liu, K. Murase, S. Inoue, C. Ge, and X. Y. Wang. Can Winds Driven by Active Galactic Nuclei Account for the Extragalactic Gamma-Ray and Neutrino Backgrounds? ApJ, 858(1):9, May 2018.

[20] M. G. Aartsen et al. Evidence for High-Energy Extraterrestrial Neutrinos at the IceCube Detector. Sci, 342:1242856, 2013. 


\section{Full Authors List: Fermi-LAT Collaboration}

M. Ajello ${ }^{1}$, L. Baldini ${ }^{2}$, J. Ballet ${ }^{3}$, G. Barbiellini ${ }^{4}$, D. Bastieri ${ }^{5}$, R. Bellazzini ${ }^{6}$, A. Berretta ${ }^{7}$, E. Bissaldi ${ }^{8}$, R. D. Blandford ${ }^{9}$, E. D. Bloom ${ }^{10}$, R. Bonino ${ }^{11}$, P. Bruel ${ }^{12}$, S. Buson ${ }^{13}$, R. A. Cameron ${ }^{14}$, D. Caprioli ${ }^{15}$, R. Caputo ${ }^{16}$, E. Cavazzuti ${ }^{17}$, G. Chartas ${ }^{18}$, S. Chen ${ }^{19}$, C. C. Cheung ${ }^{20}$, G. Chiaro ${ }^{21}$, D. Costantin ${ }^{22}$, S. Cutini ${ }^{23}$, F. D’Ammando ${ }^{24}$, P. de la Torre Luque ${ }^{25}$, F. de Palma ${ }^{26}$, A. Desai ${ }^{27}$, R. Diesing ${ }^{28}$, N. Di Lalla ${ }^{29}$, F. Dirirsa ${ }^{30}$, L. Di Venere ${ }^{31}$, A. Domínguez ${ }^{32}$, S. J. Fegan ${ }^{33}$, A. Franckowiak ${ }^{34}$, Y. Fukazawa ${ }^{35}$, S. Funk ${ }^{36}$, P. Fusco ${ }^{37}$, F. Gargano ${ }^{38}$, D. Gasparrini ${ }^{39}$, N. Giglietto ${ }^{40}$, F. Giordano ${ }^{41}$, M. Giroletti ${ }^{42}$, D. Green ${ }^{43}$, I. A. Grenier ${ }^{44}$, S. Guiriec $^{45}$, D. Hartmann ${ }^{46}$, D. Horan ${ }^{47}$, G. Jóhannesson ${ }^{48}$, C. Karwin ${ }^{49}$, M. Kerr ${ }^{50}$, M. Kovačevićéc1, M. Kuss ${ }^{52}$, S. Larsson ${ }^{53}$, L. Latronico ${ }^{54}$,

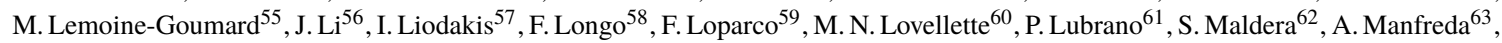
S. Marchesi ${ }^{64}$, L. Marcotulli ${ }^{65}$, G. Martí-Devesa ${ }^{66}$, M. N. Mazziotta ${ }^{67}$, I.Mereu $^{68}$, P. F. Michelson ${ }^{69}$, T. Mizuno ${ }^{70}$, M. E. Monzani ${ }^{71}$, A. Morselli ${ }^{72}$, I. V. Moskalenko ${ }^{73}$, M. Negro ${ }^{74}$, N. Omodei ${ }^{75}$, M. Orienti ${ }^{76}$, E. Orlando ${ }^{77}$, V. Paliya ${ }^{78}$, D. Paneque ${ }^{79}$, Z. Pei ${ }^{80}$, M. Persic $^{81}$, M. Pesce-Rollins ${ }^{82}$, T. A. Porter ${ }^{83}$, G. Principe ${ }^{84}$, J. L. Racusin ${ }^{85}$, S. Rainò ${ }^{86}$, R. Rando ${ }^{87}$, B. Rani ${ }^{88}$, M. Razzano ${ }^{89}$, A. Reimer ${ }^{90}$, O. Reimer ${ }^{91}$, P. M. Saz Parkinson ${ }^{92}$, D. Serini ${ }^{93}$, C. Sgrò ${ }^{94}$, E. J. Siskind ${ }^{95}$, G. Spandre ${ }^{96}$, P. Spinelli ${ }^{97}$, D. J. Suson ${ }^{98}$,

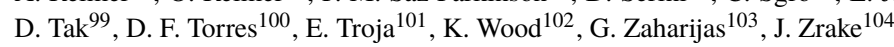

${ }^{1}$ Department of Physics and Astronomy, Clemson University, Kinard Lab of Physics, Clemson, SC 29634-0978, USA. ${ }^{2}$ Università di Pisa and Istituto Nazionale di Fisica Nucleare, Sezione di Pisa I-56127 Pisa, Italy. ${ }^{3}$ AIM, CEA, CNRS, Université Paris-Saclay, Université de Paris, F-91191 Gif-sur-Yvette, France. ${ }^{4}$ Istituto Nazionale di Fisica Nucleare, Sezione di Trieste, I-34127 Trieste, Italy. ${ }^{4}$ Dipartimento di Fisica, Università di Trieste, I-34127 Trieste, Italy. ${ }^{5}$ Istituto Nazionale di Fisica Nucleare, Sezione di Padova, I-35131 Padova, Italy. ${ }^{5}$ Dipartimento di Fisica e Astronomia "G. Galilei”, Università di Padova, I-35131 Padova, Italy. ${ }^{6}$ Istituto Nazionale di Fisica Nucleare, Sezione di Pisa, I-56127 Pisa, Italy. ${ }^{7}$ Dipartimento di Fisica, Università degli Studi di Perugia, I-06123 Perugia, Italy. ${ }^{8}$ Dipartimento di Fisica "M. Merlin" dell'Università e del Politecnico di Bari, via Amendola 173, I-70126 Bari, Italy. ${ }^{8}$ Istituto Nazionale di Fisica Nucleare, Sezione di Bari, I-70126 Bari, Italy. ${ }^{9}$ W. W. Hansen Experimental Physics Laboratory, Kavli Institute for Particle Astrophysics and Cosmology, Department of Physics and SLAC National Accelerator Laboratory, Stanford University, Stanford, CA 94305 , USA. ${ }^{10}$ W. W. Hansen Experimental Physics Laboratory, Kavli Institute for Particle Astrophysics and Cosmology, Department of Physics and SLAC National Accelerator Laboratory, Stanford University, Stanford, CA 94305, USA. ${ }^{11}$ Istituto Nazionale di Fisica Nucleare, Sezione di Torino, I-10125 Torino, Italy. ${ }^{11}$ Dipartimento di Fisica, Università degli Studi di Torino, I-10125 Torino, Italy. ${ }^{12}$ Laboratoire Leprince-Ringuet, École polytechnique, CNRS/IN2P3, F-91128 Palaiseau, France. ${ }^{13}$ Institut für Theoretische Physik and Astrophysik, Universität Würzburg, D-97074 Würzburg, Germany. ${ }^{14}$ W. W. Hansen Experimental Physics Laboratory, Kavli Institute for Particle Astrophysics and Cosmology, Department of Physics and SLAC National Accelerator Laboratory, Stanford University, Stanford, CA 94305, USA. ${ }^{15}$ Department of Astronomy and Astrophysics, University of Chicago, Chicago, IL 60637, USA. ${ }^{16}$ NASA Goddard Space Flight Center, Greenbelt, MD 20771, USA. ${ }^{17}$ Italian Space Agency, Via del Politecnico snc, 00133 Roma, Italy. ${ }^{18}$ Department of Physics and Astronomy of the College of Charleston, Charleston, SC 29424, USA. ${ }^{19}$ Istituto Nazionale di Fisica Nucleare, Sezione di Padova, I-35131 Padova, Italy. ${ }^{19}$ Department of Physics and Astronomy, University of Padova, Vicolo Osservatorio 3, I-35122 Padova, Italy. ${ }^{20}$ Space Science Division, Naval Research Laboratory, Washington, DC 20375-5352, USA. ${ }^{21}$ INAF-Istituto di Astrofisica Spaziale e Fisica Cosmica Milano, via E. Bassini 15, I-20133 Milano, Italy. ${ }^{22}$ University of Padua, Department of Statistical Science, Via 8 Febbraio, 2, 35122 Padova. ${ }^{23}$ Istituto Nazionale di Fisica Nucleare, Sezione di Perugia, I06123 Perugia, Italy. ${ }^{24}$ INAF Istituto di Radioastronomia, I-40129 Bologna, Italy. ${ }^{25}$ Dipartimento di Fisica "M. Merlin" dell'Università e del Politecnico di Bari, via Amendola 173, I-70126 Bari, Italy. ${ }^{26}$ Dipartimento di Matematica e Fisica "E. De Giorgi", Università del Salento, Lecce, Italy. ${ }^{26}$ Istituto Nazionale di Fisica Nucleare, Sezione di Lecce, I-73100 Lecce, Italy. ${ }^{27}$ Department of Physics, University of Wisconsin-Madison, Madison, WI 53706, USA. ${ }^{28}$ Department of Astronomy and Astrophysics, University of Chicago, Chicago, IL 60637, USA. ${ }^{29}$ W. W. Hansen Experimental Physics Laboratory, Kavli Institute for Particle Astrophysics and Cosmology, Department of Physics and SLAC National Accelerator Laboratory, Stanford University, Stanford, CA 94305, USA. ${ }^{30}$ Laboratoire d'Annecy-le-Vieux de Physique des Particules, Université de Savoie, CNRS/IN2P3, F-74941 Annecy-le-Vieux, France. ${ }^{31}$ Dipartimento di Fisica "M. Merlin" dell'Università e del Politecnico di Bari, via Amendola 173, I-70126 Bari, Italy. ${ }^{31}$ Istituto Nazionale di Fisica Nucleare, Sezione di Bari, I-70126 Bari, Italy. ${ }^{32}$ Grupo de Altas Energías, Universidad Complutense de Madrid, E-28040 Madrid, Spain. ${ }^{33}$ Laboratoire Leprince-Ringuet, École polytechnique, CNRS/IN2P3, F-91128 Palaiseau, France. ${ }^{34}$ Ruhr University Bochum, Faculty of Physics and Astronomy, Astronomical Institute (AIRUB), 44780 Bochum, Germany. ${ }^{35}$ Department of Physical Sciences, Hiroshima University, Higashi-Hiroshima, Hiroshima 739-8526, Japan. ${ }^{36}$ Friedrich-Alexander Universität Erlangen-Nürnberg, Erlangen Centre for Astroparticle Physics, Erwin-Rommel-Str. 1, 91058 Erlangen, Germany. ${ }^{37}$ Dipartimento di Fisica “M. Merlin" dell'Università e del Politecnico di Bari, via Amendola 173, I-70126 Bari, Italy. ${ }^{37}$ Istituto Nazionale di Fisica Nucleare, Sezione di Bari, I-70126 Bari, Italy. ${ }^{38}$ Istituto Nazionale di Fisica Nucleare, Sezione di Bari, I-70126 Bari, Italy. ${ }^{39}$ Istituto Nazionale di Fisica Nucleare, Sezione di Roma "Tor Vergata", I-00133 Roma, Italy. ${ }^{39}$ Space Science Data Center - Agenzia Spaziale Italiana, Via del Politecnico, snc, I-00133, Roma, Italy. ${ }^{40}$ Dipartimento di Fisica "M. Merlin" dell'Università e del Politecnico di Bari, via Amendola 173, I-70126 Bari, Italy. ${ }^{40}$ Istituto Nazionale di Fisica Nucleare, Sezione di Bari, I-70126 Bari, Italy. ${ }^{41}$ Dipartimento di Fisica "M. Merlin" dell’Università e del Politecnico di Bari, via Amendola 173, I-70126 Bari, Italy. ${ }^{41}$ Istituto Nazionale di Fisica Nucleare, Sezione di Bari, I-70126 Bari, Italy. ${ }^{42}$ INAF Istituto di Radioastronomia, I-40129 Bologna, Italy. ${ }^{43}$ Max-Planck-Institut für Physik, D-80805 München, Germany. ${ }^{44}$ AIM, CEA, CNRS, Université Paris-Saclay, Université de Paris, F-91191 Gif-sur-Yvette, France. ${ }^{45}$ The George Washington University, Department of Physics, 725 21st St, NW, Washington, DC 20052, USA. ${ }^{45}$ NASA Goddard Space Flight Center, Greenbelt, MD 20771, USA. ${ }^{46}$ Department of Physics and Astronomy, Clemson University, Kinard Lab of Physics, Clemson, SC 29634-0978, USA. ${ }^{47}$ Laboratoire Leprince-Ringuet, École polytechnique, CNRS/IN2P3, F-91128 Palaiseau, France. ${ }^{48}$ Science Institute, University of Iceland, IS-107 
Reykjavik, Iceland. ${ }^{48}$ Nordita, Royal Institute of Technology and Stockholm University, Roslagstullsbacken 23, SE-106 91 Stockholm, Sweden. ${ }^{49}$ Department of Physics and Astronomy, Clemson University, Kinard Lab of Physics, Clemson, SC 29634-0978, USA. ${ }^{50}$ Space Science Division, Naval Research Laboratory, Washington, DC 20375-5352, USA. ${ }^{51}$ Istituto Nazionale di Fisica Nucleare, Sezione di Perugia, I-06123 Perugia, Italy. ${ }^{52}$ Istituto Nazionale di Fisica Nucleare, Sezione di Pisa, I-56127 Pisa, Italy. ${ }^{53}$ Department of Physics, KTH Royal Institute of Technology, AlbaNova, SE-106 91 Stockholm, Sweden. ${ }^{53}$ The Oskar Klein Centre for Cosmoparticle Physics, AlbaNova, SE-106 91 Stockholm, Sweden. ${ }^{53}$ School of Education, Health and Social Studies, Natural Science, Dalarna University, SE-791 88 Falun, Sweden. ${ }^{54}$ Istituto Nazionale di Fisica Nucleare, Sezione di Torino, I-10125 Torino, Italy. ${ }^{55}$ Centre d'Études Nucléaires de Bordeaux Gradignan, IN2P3/CNRS, Université Bordeaux 1, BP120, F-33175 Gradignan Cedex, France. ${ }^{56}$ Department of Astronomy, School of Physical Sciences, University of Science and Technology of China, Hefei, Anhui 230026, China. ${ }^{57}$ Finnish Centre for Astronomy with ESO (FINCA), University of Turku, FI-21500 Piikiiö, Finland. ${ }^{58}$ Istituto Nazionale di Fisica Nucleare, Sezione di Trieste, I-34127 Trieste, Italy. ${ }^{58}$ Dipartimento di Fisica, Università di Trieste, I-34127 Trieste, Italy. ${ }^{59}$ Dipartimento di Fisica "M. Merlin" dell'Università e del Politecnico di Bari, via Amendola 173, I-70126 Bari, Italy. ${ }^{59}$ Istituto Nazionale di Fisica Nucleare, Sezione di Bari, I-70126 Bari, Italy. ${ }^{60}$ Space Science Division, Naval Research Laboratory, Washington, DC 20375-5352, USA. ${ }^{61}$ Istituto Nazionale di Fisica Nucleare, Sezione di Perugia, I-06123 Perugia, Italy. ${ }^{62}$ Istituto Nazionale di Fisica Nucleare, Sezione di Torino, I-10125 Torino, Italy. ${ }^{63}$ Università di Pisa and Istituto Nazionale di Fisica Nucleare, Sezione di Pisa I-56127 Pisa, Italy. ${ }^{64}$ INAF - Osservatorio di Astrofisica e Scienza dello Spazio di Bologna, Via Piero Gobetti, 93/3, 40129, Bologna, Italy. ${ }^{65}$ Department of Physics and Astronomy, Clemson University, Kinard Lab of Physics, Clemson, SC 29634-0978, USA. ${ }^{66}$ Institut für Astro- und Teilchenphysik, Leopold-Franzens-Universität Innsbruck, A-6020 Innsbruck, Austria. ${ }^{67}$ Istituto Nazionale di Fisica Nucleare, Sezione di Bari, I-70126 Bari, Italy. ${ }^{68}$ Dipartimento di Fisica, Università degli Studi di Perugia, I-06123 Perugia, Italy. ${ }^{68}$ Istituto Nazionale di Fisica Nucleare, Sezione di Perugia, I-06123 Perugia, Italy. ${ }^{69}$ W. W. Hansen Experimental Physics Laboratory, Kavli Institute for Particle Astrophysics and Cosmology, Department of Physics and SLAC National Accelerator Laboratory, Stanford University, Stanford, CA 94305, USA. ${ }^{70}$ Hiroshima Astrophysical Science Center, Hiroshima University, Higashi-Hiroshima, Hiroshima 739-8526, Japan. ${ }^{71}$ W. W. Hansen Experimental Physics Laboratory, Kavli Institute for Particle Astrophysics and Cosmology, Department of Physics and SLAC National Accelerator Laboratory, Stanford University, Stanford, CA 94305, USA. ${ }^{72}$ Istituto Nazionale di Fisica Nucleare, Sezione di Roma "Tor Vergata", I-00133 Roma, Italy. ${ }^{73}$ W. W. Hansen Experimental Physics Laboratory, Kavli Institute for Particle Astrophysics and Cosmology, Department of Physics and SLAC National Accelerator Laboratory, Stanford University, Stanford, CA 94305, USA. ${ }^{74}$ Center for Research and Exploration in Space Science and Technology (CRESST) and NASA Goddard Space Flight Center, Greenbelt, MD 20771, USA. ${ }^{74}$ Department of Physics and Center for Space Sciences and Technology, University of Maryland Baltimore County, Baltimore, MD 21250, USA. ${ }^{75}$ W. W. Hansen Experimental Physics Laboratory, Kavli Institute for Particle Astrophysics and Cosmology, Department of Physics and SLAC National Accelerator Laboratory, Stanford University, Stanford, CA 94305, USA. ${ }^{76}$ INAF Istituto di Radioastronomia, I-40129 Bologna, Italy. ${ }^{77}$ Istituto Nazionale di Fisica Nucleare, Sezione di Trieste, and Università di Trieste, I-34127 Trieste, Italy. ${ }^{77}$ W. W. Hansen Experimental Physics Laboratory, Kavli Institute for Particle Astrophysics and Cosmology, Department of Physics and SLAC National Accelerator Laboratory, Stanford University, Stanford, CA 94305, USA. ${ }^{78}$ Aryabhatta Research Institute of Observational Sciences (ARIES), Manora Peak, Nainital-263 129, Uttarakhand, India. ${ }^{78}$ Deutsches Elektronen Synchrotron DESY, D-15738 Zeuthen, Germany. ${ }^{79}$ Max-Planck-Institut für Physik, D-80805 München, Germany. ${ }^{80}$ Dipartimento di Fisica e Astronomia "G. Galilei", Università di Padova, I-35131 Padova, Italy. ${ }^{81}$ Istituto Nazionale di Fisica Nucleare, Sezione di Trieste, I-34127 Trieste, Italy. ${ }^{81}$ Osservatorio Astronomico di Trieste, Istituto Nazionale di Astrofisica, I-34143 Trieste, Italy. ${ }^{82}$ Istituto Nazionale di Fisica Nucleare, Sezione di Pisa, I-56127 Pisa, Italy. ${ }^{83}$ W. W. Hansen Experimental Physics Laboratory, Kavli Institute for Particle Astrophysics and Cosmology, Department of Physics and SLAC National Accelerator Laboratory, Stanford University, Stanford, CA 94305, USA. ${ }^{84}$ Dipartimento di Fisica, Università di Trieste, I-34127 Trieste, Italy. ${ }^{84}$ Istituto Nazionale di Fisica Nucleare, Sezione di Trieste, I-34127 Trieste, Italy. ${ }^{84}$ INAF Istituto di Radioastronomia, I-40129 Bologna, Italy. ${ }^{85}$ NASA Goddard Space Flight Center, Greenbelt, MD 20771, USA. ${ }^{86}$ Dipartimento di Fisica "M. Merlin" dell’Università e del Politecnico di Bari, via Amendola 173, I-70126 Bari, Italy. ${ }^{86}$ Istituto Nazionale di Fisica Nucleare, Sezione di Bari, I-70126 Bari, Italy. ${ }^{87}$ Department of Physics and Astronomy, University of Padova, Vicolo Osservatorio 3, I-35122 Padova, Italy. ${ }^{87}$ Istituto Nazionale di Fisica Nucleare, Sezione di Padova, I-35131 Padova, Italy. ${ }^{87}$ Center for Space Studies and Activities "G. Colombo", University of Padova, Via Venezia 15, I-35131 Padova, Italy. ${ }^{88}$ Korea Astronomy and Space Science Institute, 776 Daedeokdae-ro, Yuseong-gu, Daejeon 30455, Korea. ${ }^{88}$ NASA Goddard Space Flight Center, Greenbelt, MD 20771, USA. ${ }^{88}$ Department of Physics, American University, Washington, DC 20016, USA. ${ }^{89}$ Istituto Nazionale di Fisica Nucleare, Sezione di Pisa, I-56127 Pisa, Italy. ${ }^{89}$ Funded by contract FIRB-2012-RBFR12PM1F from the Italian Ministry of Education, University and Research (MIUR). ${ }^{90}$ Institut für Astro- und Teilchenphysik, Leopold-Franzens-Universität Innsbruck, A-6020 Innsbruck, Austria. ${ }^{90}$ W. W. Hansen Experimental Physics Laboratory, Kavli Institute for Particle Astrophysics and Cosmology, Department of Physics and SLAC National Accelerator Laboratory, Stanford University, Stanford, CA 94305, USA. ${ }^{91}$ Institut für Astro- und Teilchenphysik, Leopold-Franzens-Universität Innsbruck, A-6020 Innsbruck, Austria. ${ }^{92}$ Santa Cruz Institute for Particle Physics, Department of Physics and Department of Astronomy and Astrophysics, University of California at Santa Cruz, Santa Cruz, CA 95064, USA. ${ }^{92}$ Department of Physics, The University of Hong Kong, Pokfulam Road, Hong Kong, China. ${ }^{92}$ Laboratory for Space Research, The University of Hong Kong, Hong Kong, China. ${ }^{93}$ Dipartimento di Fisica "M. Merlin" dell'Università e del Politecnico di Bari, via Amendola 173, I-70126 Bari, Italy. ${ }^{94}$ Istituto Nazionale di Fisica Nucleare, Sezione di Pisa, I-56127 Pisa, Italy. ${ }^{95}$ NYCB Real-Time Computing Inc., Lattingtown, NY 11560-1025, USA. ${ }^{96}$ Istituto Nazionale di Fisica Nucleare, Sezione di Pisa, I-56127 Pisa, Italy. ${ }^{97}$ Dipartimento di Fisica "M. Merlin" dell'Università e del Politecnico di Bari, via Amendola 173, I-70126 Bari, Italy. ${ }^{97}$ Istituto Nazionale di Fisica Nucleare, Sezione di Bari, I-70126 Bari, Italy. ${ }^{98}$ Purdue University Northwest, Hammond, IN 46323, USA. ${ }^{99}$ Department of Physics, University of Maryland, College Park, MD 20742, USA. ${ }^{99}$ NASA Goddard Space Flight Center, Greenbelt, MD 20771, USA. ${ }^{100}$ Institute of Space Sciences (ICE, CSIC), Campus UAB, Carrer de Magrans s/n, E-08193 Barcelona, 
Spain; and Institut d'Estudis Espacials de Catalunya (IEEC), E-08034 Barcelona, Spain. ${ }^{100}$ Institució Catalana de Recerca i Estudis Avançats (ICREA), E-08010 Barcelona, Spain. ${ }^{101}$ NASA Goddard Space Flight Center, Greenbelt, MD 20771, USA. ${ }^{101}$ Department of Astronomy, University of Maryland, College Park, MD 20742, USA. ${ }^{102}$ Praxis Inc., Alexandria, VA 22303, resident at Naval Research Laboratory, Washington, DC 20375, USA. ${ }^{103}$ Istituto Nazionale di Fisica Nucleare, Sezione di Trieste, and Università di Trieste, I-34127 Trieste, Italy. ${ }^{103}$ Center for Astrophysics and Cosmology, University of Nova Gorica, Nova Gorica, Slovenia. ${ }^{104}$ Department of Physics and Astronomy, Clemson University, Kinard Lab of Physics, Clemson, SC 29634-0978, USA. 